\title{
Probing Reheating Temperature with primordial Gravitational Waves: with application to Gauss-Bonnet inflation
}

\author{
Gansukh Tumurtushaa*† \\ Center for Theoretical Physics of the Universe, Institute for Basic Science, Daejeon 34051, \\ Korea \\ E-mail: gansuhmgl@ibs.re.kr
}

\section{Seoktae Koh}

Department of Science Education, Jeju National University, Jeju, 63243, Korea

E-mail: kundol.kohejejunu.ac.kr

\section{Bum-Hoon Lee}

Center for Quantum Spacetime, Sogang University, Seoul 121-742, Korea

Department of Physics, Sogang University, Seoul 121-742, Korea

E-mail: bhl@sogang.ac.kr

\begin{abstract}
We estimate the reheating temperature of the universe after Gauss-Bonnet (GB) inflation by using the primordial gravitational wave (PGW). Inflationary tensor power spectrum has a blue tilt $\left(n_{T}>0\right)$ for the selected model of inflation; hence, the energy spectrum of the PGW significantly enhances. As a result, the PGW spectrum induced by GB inflation is detectable by future concepts of GW detectors. We further perform analyses on the reheating parameters including the temperature, the equation of state, and the number of e-folds. Our results imply that the Gauss-Bonnet term plays an important role not only during inflation but also during reheating whether the process is instantaneous or lasts for a certain number of e-folds until it thermalizes and eventually completes. This presentation is based on our previous works [2].
\end{abstract}

The 39th International Conference on High Energy Physics (ICHEP2018)

4-11 July, 2018

Seoul, Korea

\footnotetext{
*Speaker.

${ }^{\dagger}$ corresponding author
} 


\section{Introduction}

As a result of an accelerated expansion, cosmic inflation predicts nearly scale-invariant spectrum of the cosmic microwave (CMB) background anisotropies and the generation of the PGW, and it provides seeds to the large-scale structure of the universe [1]. The temperature of the universe during inflation became almost zero; hence, it is necessary to reheat the universe after inflation came to the end. A transition epoch between the end of inflation and the beginning of the radiationdominated era is known as the reheating epoch. The physics of reheating is highly uncertain and unconstrained. There is no direct cosmological observation traceable this period. Thus, the physics of reheating depends on inflation models. In this work, therefore, we consider inflationary models with GB term to estimate the reheating temperature [2]. The GB term is previously studied in the context of inflation, reheating, and PGW [3]. Since the universe is transparent to the GWs up to Planck era. The detection of the PGW background by a future observation would open up a new window in exploring the early universe, particularly the reheating epoch $[4,5]$. Thus, in this work, we use the PGW as a probe to study the reheating era. This letter is organized as follows. In Sec. 2, we introduce our inflation model with the GB term and calculate the energy spectrum of the PGW. Motivated by the fact that the reheating temperature could be determined by the detection of the PGW background, we perform analyses on the reheating temperature in Sec. 3. The conclusion of the present work is presented in Sec. 4.

\section{PGW spectrum induced by inflationary models with GB term}

The action we consider is

$$
S=\int d^{4} x \sqrt{-g}\left[\frac{1}{2 \kappa^{2}} R-\frac{1}{2} g^{\mu v} \partial_{\mu} \phi \partial_{\nu} \phi-V(\phi)-\frac{1}{2} \xi(\phi) R_{\mathrm{GB}}^{2}\right],
$$

where $R_{\mathrm{GB}}^{2}=R_{\mu v \rho \sigma} R^{\mu v \rho \sigma}-4 R_{\mu v} R^{\mu v}+R^{2}$ is known as the GB term and $\kappa^{2}=8 \pi G=M_{\mathrm{pl}}^{-2}$ is the reduced Planck mass. The potential and the coupling function are respectively given by

$$
V(\phi)=\frac{1}{\kappa^{4}}[\tanh (\kappa \phi)+\sqrt{\mu} \operatorname{sech}(\kappa \phi)]^{2}, \quad \xi(\phi)=\frac{3\left[\sinh ^{2}(\kappa \phi)-\frac{1}{\sqrt{\mu}} \sinh (\kappa \phi)\right]}{4[\sqrt{\mu}+\sinh (\kappa \phi)]^{2}},
$$

where $\mu>0$. The PGWs are characterized by a tensor part of the metric fluctuations: $d s^{2}=$ $a^{2}(\tau)\left[-d \tau^{2}+\left(\delta_{i j}+h_{i j}\right) d x^{i} d x^{j}\right]$, where $h_{i j}$ is symmetric under the exchange of indices and satisfies $\partial_{i} h^{i j}=0, \delta^{i j} h_{i j}=0$. The strength of the PGWs is determined by their energy spectrum

$$
h_{0}^{2} \Omega_{G W}=\frac{3 h_{0}^{2}}{32 \pi^{2} H_{0}^{2} \tau_{0}^{4} f^{2}} \Omega_{m}^{2} \mathscr{T}_{1}^{2}\left(\frac{f}{f_{\mathrm{eq}}}\right) \mathscr{T}_{2}^{2}\left(\frac{f}{f_{\mathrm{th}}}\right) r \mathscr{P}_{S}\left(\frac{f}{f_{*}}\right)^{n_{T}},
$$

where $H_{0}=100 h_{0} \mathrm{~km} \mathrm{~s}^{-1} \mathrm{Mpc}^{-1}$ with $h_{0}=0.6731$ [1] is the Hubble constant, $\tau_{0}$ is conformal time, and $r$ is the tensor-to-scalar ratio. The scalar power spectrum is well measured as $\ln \left(10^{10} \mathscr{P}_{S}\right)=3.089_{-0.027}^{+0.024}$ at the pivot scale $k_{*}=0.05 \mathrm{Mpc}^{-1}$ [1]. For our model in Eq. (2.2), $n_{T}=2 \mu\left(N_{*}-1\right) /\left[\left(N_{*}+\mu\right)\left(N_{*}^{2}+\mu\right)\right]$ and $r=8 /\left(N_{*}^{2}+\mu\right)$ with $N_{*}=2 /\left(1-n_{S}\right)-\mu$ [2]. The transfer function $\mathscr{T}^{2}(k)$ reflects the evolution of GWs after horizon reentry. If modes that reenter the horizon during the matter dominated era, 
$\mathscr{T}^{2} \sim 9 /(k \tau)^{4}$ [4]. For modes that reentered the horizon near the matter and radiation equality and the end of reheating, we get $\mathscr{T}_{1}^{2}\left(x_{\mathrm{eq}}\right)=1+1.65 x_{\mathrm{eq}}+1.92 x_{\mathrm{eq}}^{2}$ and $\mathscr{T}_{2}^{2}\left(x_{\mathrm{th}}\right)=\left(1-0.23 x_{\mathrm{th}}^{3 / 2}+0.58 x_{\mathrm{th}}^{2}\right)^{-1}$, respectively, where $x_{\text {eq }}=k / k_{\text {eq }}$ with $k_{\text {eq }}=7.3 \times 10^{-2} \Omega_{m} h_{0}^{2} \mathrm{Mpc}^{-1}$ at the equality and $x_{\text {th }}=k / k_{\text {th }}$ with $k_{\text {th }}=$ $1.7 \times 10^{13} \mathrm{Mpc}^{-1}\left(g_{* s}\left(T_{\mathrm{th}}\right) / 106.75\right)^{1 / 6}\left(T_{\mathrm{th}} / 10^{6} \mathrm{GeV}\right)$ at the time of reheating [2]. In Fig. 1(a), we plot Eq. (2.3) as a function of frequency for varying $\mu$ and $T_{\text {th }}$. The result shows that the PGW from Gauss-Bonnet inflation can be probed by DECIGO [6].

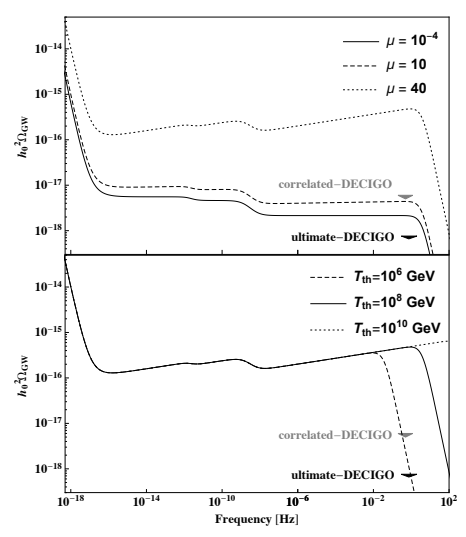

(a) $h_{0}^{2} \Omega_{\mathrm{GW}}$ as a function of frequency $f$.

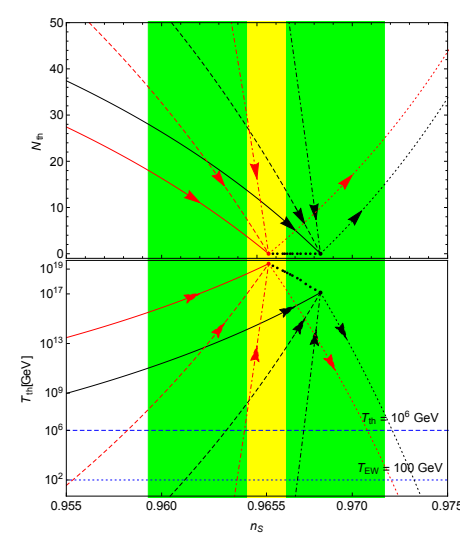

(b) The $n_{s}$ dependence on $N_{\mathrm{th}}$ and $T_{\mathrm{th}}$.

Figure 1: (Left) We set $T_{\text {th }}=10^{8} \mathrm{GeV}$ in the upper panel and $\mu=40$ in the bottom panel. (Right) From left to right (solid to dotted), $\omega_{\text {th }}=-1 / 3,0,1 / 4,1$. The black dots reaching the red one indicate the instantaneous reheating $\left(N_{\text {th }}=0\right)$. The $\mu$ value increases between $10^{-4} \leq \mu \leq 0.3517$ from the black lines to the red ones. $N_{*}$ increases along the direction of the arrow. The shaded regions correspond to the $1 \sigma$ range of the current (green) Planck [1] and the future (yellow) CMB experiments [8] with the central value at $n_{S}=0.9655$ from Planck data. The horizontal lines at $T_{\mathrm{EW}}=10^{2} \mathrm{GeV}$ and $T_{\mathrm{th}}=10^{6} \mathrm{GeV}$ indicate the electroweak scale and the lower bound from the PGW detection by DECIGO, respectively.

\section{Constraints on the reheating temperature}

If the PGWs from inflation is detected, the temperature $T_{\text {th }}$ of reheating can be determined by the frequency at which it is detected [5], $f_{\mathrm{th}} \simeq 0.27 \times 10^{13} \mathrm{Mpc}^{-1}\left(g_{* s}\left(T_{\mathrm{th}}\right) / 106.75\right)^{1 / 6}\left(T_{\mathrm{th}} / 10^{6} \mathrm{GeV}\right)$. In the following, we estimate $T_{\text {th }}$ by using the energy spectrum of the PGW induced by Gauss-Bonnet inflation. $T_{\text {th }}$ determines the energy density $\rho_{\text {th }}$ at the end of reheating: $\rho_{\text {th }}=\frac{\pi^{2}}{30} g_{*}\left(T_{\text {th }}\right) T_{\text {th }}^{4}$, where $g_{*}\left(T_{\mathrm{th}}\right)$ is the number of relativistic degrees of freedom at the end of reheating. Furthermore, $\rho_{\mathrm{th}}$ is related to the energy density at the end of inflation via $\rho_{\mathrm{th}}=\rho_{\mathrm{end}} e^{-3\left(1+\omega_{\mathrm{th}}\right) N_{\mathrm{th}}}$, where $\omega_{\mathrm{th}}$ and $N_{\mathrm{th}}$ are the equation of state and the duration of reheating, respectively. $N_{\text {th }}$ is given by [7],

$$
N_{\mathrm{th}}=\frac{4}{3 \omega_{\mathrm{th}}-1}\left[\ln \left(\frac{k}{a_{0} T_{0}}\right)+\frac{1}{3} \ln \left(\frac{11 g_{* s}}{43}\right)+\frac{1}{4} \ln \left(\frac{30 \lambda_{\mathrm{end}}}{\pi^{2} g_{*}}\right)+\frac{1}{4} \ln \left(\frac{V_{\mathrm{end}}}{H_{*}^{4}}\right)+N_{*}\right],
$$

where $\omega_{\text {th }} \neq 1 / 3$ and $\rho_{\text {end }}=\lambda_{\text {end }} V_{\text {end }}$ are assumed. The subscripts " 0 " and " $*$ " denote the current and the horizon crossing values of each quantity, respectively. Thus, $T_{\text {th }}$ becomes

$$
T_{\mathrm{th}}=\left(\frac{30 \lambda_{\mathrm{end}} V_{\mathrm{end}}}{\pi^{2} g_{*}}\right)^{\frac{1}{4}} e^{-\frac{3}{4}\left(1+\omega_{\mathrm{th}}\right) N_{\mathrm{th}}} .
$$


Here, the potential energy $V_{\text {end }}$ and the effective ratio of kinetic energy to potential energy $\lambda_{\text {end }}$ at the end of inflation are respectively obtained as

$$
V_{\text {end }}=\frac{1}{\kappa^{4}} \frac{(\mu+x)^{2}}{1+x^{2}}, \quad \lambda_{\text {end }}=\frac{6 \mu^{3 / 2}\left(x^{2}+1\right)^{2}(\sqrt{\mu}+x)}{6 \mu^{2}\left(x^{2}+1\right)^{2}-3 \mu\left(x^{2}+3\right)+\sqrt{\mu} x\left(5 x^{2}+2\right)+2 x^{2}+6 \mu^{3 / 2} x\left(x^{4}+4 x^{2}+3\right)-1},
$$

where $x=-\sqrt{\mu} / 3\left[1+(\mu-6)\left(2 / x_{1}\right)^{1 / 3}+\left(2 / x_{1}\right)^{-1 / 3} / \mu\right]$, and $x_{1}=2 \mu^{3}+9 \mu^{2}-27 \mu+\sqrt{27\left(4 \mu^{5}-17 \mu^{4}+14 \mu^{3}+27 \mu^{2}\right)}$. Using Eqs. (3.1) and (3.2), we plot $N_{\text {th }}$ and $T_{\text {th }}$ in Fig. 1(b) as functions of $n_{S}$ for varying $\mu$ and $\omega_{\text {th }}$. Our result shows that $T_{\text {th }}$ significantly enhances due to the presence of the GB term.

\section{Conclusion}

We have estimated the reheating temperature of the universe both analytically and numerically. In our analyses, we considered inflationary models with Gauss-Bonnet term. The main results of our work are presented in Eqs. (3.1) and (3.2) and in Fig. 1. The lower limit of reheating temperature is determined by the detection of the PWG spectrum and is $T_{\text {th }} \geq 10^{6} \mathrm{GeV}$ for our model; see Fig. 1(a). We showed that $T_{\text {th }}$ is significantly enhanced due to the presence of the GB term. However, the upper limit is determined by the observational constraint on $n_{S}$; see Fig. 1(b). Since the $n_{S}$ value is well measured by CMB observation, it can reduce the parameter space; hence, it indirectly puts constraints on $T_{\text {th }}$. Thus, we conclude that the GB term plays an important role during reheating whether the process is instantaneous or lasts for certain $e$-folds until it completes.

\section{Acknowledgments}

The work of G. T was supported by IBS under the project code, IBS-R018-D1. S. Koh was supported by Basic Science Research Program through the NRF of Korea funded by the Ministry of Education (No. NRF2016R1D1A1B04932574). B. H. Lee was supported by Basic Science Research Program through the NRF of Korea funded by the Ministry of Education (No.

NRF2018R1D1A1B07048657).

\section{References}

[1] P. A. R. Ade et al. [Planck Collaboration], Astron. Astrophys. 594, A20 (2016).

[2] S. Koh, B. H. Lee and G. Tumurtushaa, Phys. Rev. D 98, 103511 (2018); S. Koh, B. H. Lee, and G. Tumurtushaa, Phys. Rev. D 95, 123509 (2017); S. Koh, B. H. Lee, W. Lee, and G. Tumurtushaa, Phys. Rev. D 90, 063527 (2014).

[3] M. Satoh and J. Soda, J. Cosmol. Astropart. Phys. 09 (2008) 019; Z. K. Guo and D. J. Schwarz, Phys. Rev. D 81, 123520 (2010); C. van de Bruck, K. Dimopoulos, and C. Longden, Phys. Rev. D 94, 023506 (2016);

[4] M. S. Turner, M. J. White and J. E. Lidsey, Phys. Rev. D 48, 4613 (1993);

[5] K. Nakayama, S. Saito, Y. Suwa and J. Yokoyama, JCAP 0806, 020 (2008);

[6] S. Kawamura et al., Classical Quantum Gravity 28, 094011 (2011).

[7] L. Dai, M. Kamionkowski and J. Wang, Phys. Rev. Lett. 113, 041302 (2014);

[8] L. Amendola et al. [Euclid Theory Working Group Collaboration], Living Rev. Rel. 16, 6 (2013); P. Andre et al. [PRISM Collaboration], arXiv:1306.2259. 